\title{
Persistence and Ontological Pluralism
}

\author{
PIERRE GRENON / BARRY SMITH \\ Saarbrücken, Genève / Saarbrücken, Buffalo
}

Preprint version of paper to appear in C. Kanzian (ed.), Persistence, New York: Springer, 2007

\section{Introduction}

Consider Fritz, who is nowadays a happy resident of Saarbrücken. On sunny Saturdays, Fritz has the habit of barbecuing Bratwurst with his friends in his yard and going thereafter to his local, the Nächstestrasse Biergarten, 'NB' for short. Fritz may have a couple of beers while eating his Bratwurst at home, but nothing which compares to the amount he will drink thereafter at the beer garden. That is why he always goes by foot: he knows that his Blood Alcohol Content, 'BAC' hereafter, will not allow him to drive his car back home when the time comes to leave. We can break down Fritz's typical Saturday in the following way:

i) Fritz starts his day with a very low BAC.

ii) Fritz stays at home and eats and drinks reasonably during the afternoon. His BAC rises significantly but not dramatically.

iii) Fritz goes to NB, where he drinks a less reasonable amount of beer, increasing dramatically his BAC.

iv) Fritz goes back home, sleeps and lets his BAC level fall back to a normal level.

What have we done here? We have divided up a day according to Fritz's activities and the changes in Fritz and his BAC. We have mentioned several entities, either by name or by description; for instance, Fritz, the beer garden, Fritz's BAC, Fritz's home, Fritz's activities on this Saturday, which break down into an afternoon at home, a walk to the NB, a sojourn at NB, a walk back. These activities themselves break down into various sub-activities of Bratwurst eating, beer drinking, step-taking, and many others.

Not all of these entities are on a par. Surely, all those we have mentioned exist in time. It seems also that they are all entities which last for more than a mere instant. They are temporally extended. But there is a way in which some differ from and a way some resemble each other. 
By the end of the day, Fritz has changed in many ways. He has changed because at the start of the day he had neither yet eaten any Bratwurst nor drunk any beer nor walked to the NB, etc. In particular, he has changed because yet another entity, namely his BAC, has changed. In fact the description of Fritz's typical Saturday was in part an account of the changes in his BAC, and hence in himself, over such a day. The Bratwurst, too, has changed: each sausage went from raw to cooked to going out of existence as a result of being eaten. There have been many qualitative changes (from raw to cooked, from ajeun to repus, from sober to intoxicated) and many spatial changes also (from the fridge to the barbecue to the bun, from the kitchen to the garden, from home to NB, from NB to home).

Fritz, his BAC and his tiredness are entities which persist in time and remain numerically identical through the various changes which they undergo. Fritz is the same entity before, during, and after eating Bratwurst and over the whole day, although he has changed during this time. Similarly, his BAC has changed, but it is the same entity which has gone from low to mild and from mild to high on the alcoholemic scale. Fritz has gone from rested to tired, but it is still always Fritz, through and through, one and identical.

Some of the other entities which we have mentioned are in fact some of these very changes. For instance, Fritz's walk from home to NB is one of Fritz's changes in location. Changes are processes. Taking a step, drinking a beer, spending an evening at the beer garden are examples of entities which are temporally extended. But in contradistinction to Fritz and his BAC, they do not change. It is not that Fritz's spending an evening at the beergarden is first a sitting, then a beer drinking, and so on and so forth. The spending of the evening lasts the whole evening, the beer drinking lasts a beer, so to speak. The spending of the evening at NB is a complex entity made of many other entities which exist only during some part of the period during which the whole sojourn exists. Thus there is that part of the evening during which Fritz is drinking his sixth beer at the NB.

Entities of this second kind do not change, because they are themselves changes, and they are made up of successive (temporal) parts which are also changes.

Fritz himself, however, is not made up of temporal parts. He exists throughout the whole evening (and before and after, of course), and there is no entity which would be the transient proxy of Fritz during which he is involved in this or that activity, or during which he or his BAC sustains this or that change. There is no shorter-lived part of Fritz whose existence 
is restricted to these periods (Inwagen 2000). Fritz's evening is not a part of Fritz, any more than is Fritz's youth or Fritz's passage down the birth canal. Rather, these are parts of Fritz's life.

In this paper we aim to provide the ontological grounds for an adequate account of persistence. We defend a perspectivalist, or moderate pluralist, position, according to which some aspects of reality can be accounted for in ontological terms only via partial and mutually complementary ontologies, each one of which captures some relevant aspect of reality. Our thesis here is that this is precisely the sort of ontological account that is needed for the understanding of persistence.

Our brand of moderate pluralism is a generic doctrine, which has been applied to the problem of granularity (Smith and Brogaard 2003; Bittner and Smith 2003). Here we will present reasons for adopting the doctrine also as providing a solution to the problem of persistence. We sketch the main categories of possible ontologies for endurance and perdurance without, however, arguing for these in any detail. In particular we do not address the topic of substantivalism versus relationalism about space, time or spacetime in detail, because we think that our approach is compatible with each major variant position on such topics. What we do address in detail is the need for two independent ontologies in any account of persistence.

\section{Two Sorts of Entities}

According to our account of Fritz and of certain portions of his life, there are entities that have continuous existence and a capacity to persist selfidentically through time even while undergoing different sorts of changes. These entities, sometimes called 'continuants', come in several kinds; but for the purposes of this paper we will give only a sketch of the most relevant types. On the one hand there are substances and other substantial entities, which are all bearers of property particulars, and on the other hand there are these property particulars themselves - Fritz's present thirst, for example, or his BAC (Smith 1990). Substances are those material entities that enjoy a certain rounded-offness or natural completeness (Smith and Broogard 2003). In addition to artefacts such as mugs and sausages, organisms are the prototypical examples of substances. The wider category of substantial entities embraces not only substances in this sense, but also 
their parts and aggregates, the parts and aggregates of these parts and aggregates, and so forth. Substantial entities are not dependent for their continued existence upon other entities; they are the bearers of qualities, and they are subject to qualitative change. Property particulars are endurants requiring a basis in substantial entities in order to exist; they inhere in the latter and this inherence is a form of existential dependence (Husserl 1913/21; Simons 1987). Examples of property particulars include: particularized qualities (the colour of this sausage, the ambient temperature in the NG), functions (for example, the function of this barbecue: to cook food), roles (as customer, as waiter), dispositions (of vegetables, that they are prone to rotting), and so on. Finally, for the sake of expository simplicity, we embrace here a so-called absolutist view of space, sometimes called a container view. According to this view, spatial regions are entities in their own right, which are endurants of such a sort that other endurants can be located at or in them.

In addition, the world contains entities traditionally called 'occurrents' - entities which occur, which happen to continuants, more familiarly referred to as processes, events, activities, changes, happenings. Occurrents are entities which unfold themselves through a period of time or they are the temporal boundaries of such entities. The former are all bound in time in the way described by Zemach (1970). This means that each portion of the time during which an occurrent occurs can be associated with a corresponding temporal portion of that occurrent. This is because occurrents exist only in their successive temporal parts or phases. Some of the latter for example, beginnings and endings (the initial and terminal boundaries of processes) - are instantaneous. Following Ingarden (1964), we shall reserve the term 'process' for temporally extended occurrents, and 'event' for their instantaneous boundaries (which we take to include also the crossings of transition thresholds within processes). Examples are: the beginning of Fritz's walk; Fritz's becoming 19 years of age; the detaching of a portion of sausage as a result of Fritz taking a bite out of it; the ceasing to exist of an electron as a result of collision with a positron. On the occurrent side of our ontology we include also the temporal and spatiotemporal regions at which other occurrents are located.

Prima facie, the view which seems hereby implied, a view which we believe is in close conformity with common sense, is that of a bicategorial ontology, embracing both continuants and occurrents as entities with equal standing. Common sense, however, is not for us an ultimate justification. Indeed, although we will defend a view that is close to common sense in 
that it recognizes virtually the same entities, we think nonetheless that a bicategorial ontology is untenable.

In mainstream analytical philosophy, the name of the game when it comes to the problem of persistence in time is eliminativism. This forces us to choose between either an ontology of continuants, so-called 'threedimensionalism', or an ontology of occurrents, so-called 'fourdimensionalism'. Following Lewis (1986), the view that continuants are threedimensional is sometimes called 'endurantism' (and the way they exist in time is to endure), while the view that continuants are fourdimensional is sometimes called 'perdurantism' (and the way they exist in time is to perdure). From our perspective, however, there are in fact four available alternatives from which to choose:

1) threedimensionalism, according to which the correct ontology for handling persistence is a monocategorial ontology of continuants;

2) fourdimensionalism, according to which the correct ontology for handling persistence is a monocategorial ontology of occurrents;

3) bicategorialism, according to which the correct ontology for handling persistence is a bicategorial ontology of continuants and occurrents;

4) two-ontology moderate pluralism, according to which there is no correct and adequate way of handling persistence within a single ontology, but rather two ontologies are needed, which must somehow be made to work in tandem.

While each of the eliminativist strategies is found wanting not only when measured against common sense but also when measured against the increasingly important needs of ontology applications, for example in the field of biomedicine (Grenon, Smith and Goldberg 2004), bicategorialism leads to contradictions. These problems do not, however, arise on the fourth option, which is thus the option we favor.

\section{Rejection of Solutions based on Single Ontologies}

\subsection{Rejection of Bicategorialism}

The reasons for rejecting bicategorialism have to do with the fact that each of the two categories of continuant and of occurrent requires an ontology with a characteristic which conflicts with a characteristic of the ontology required by the other category. There is no single ontology countenancing 
both continuants and occurrents because the proper treatment of these entities requires different and conflicting treatments of time (Merricks 1995). Reasons for this have to do with the fact that under the view of time required for the proper handling of occurrents, continuants possess incompatible determinations, the countenancing of which leads to contradictions. On the other hand, as we shall see shortly, under the view of time required by an ontology which recognizes continuants, occurrents simply cannot be countenanced.

Strictly speaking, we should not say that continuants exist in time, because this expression suggests that continuants are temporal entities which belong to an ontology in which time is an entity in its own right. However, time itself is not a continuant, and thus it lies beyond the scope of the continuant ontology. Persisting continuants exist at multiple successive times, and they persist from one time to the next. From the point of view of ontology, however, they have to be taken, so to speak, one time at a time. We could say that they exist over a time in the sense just explained. But because time does not belong to an ontology of continuants, the contrast between existing in time and over a time or at multiple times cannot be expressed using the terms of such an ontology. We can (at the risk of some metaphor) look upon time as a dimension of location along which continuants can move; but that dimension is ipso facto extraneous to the realm of continuants. From the standpoint of an ontology of continuants, to appeal to time would be at best to appeal to an abstraction.

Taking temporality into account on the side of continuants thus requires a multiplicity of time-indexed ontologies of continuants corresponding to the points along this temporal dimension. There is only one Fritz; but to do justice to his persistence through time requires a multiplicity of continuant

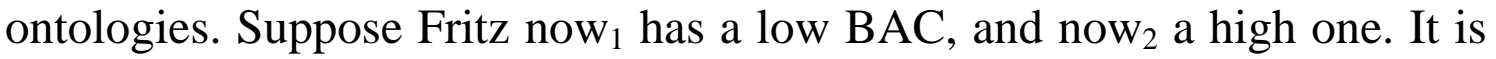
not possible to account for the change in Fritz if the Fritz who exists now 1 is seen as an entity existentially on a par with the Fritz who exists now 2 . This is because the two entities would have contradictory properties in virtue of the fact that Fritz has changed (Lowe 2002). It is in order to do justice to this fact that continuant ontologies like the one we favour see continuants as existing always at a time. The ontology of continuants is in a sense always exemplified in a synchronic fashion - it is exemplified now at this moment, now at this moment. There is therefore a need for a multiplicity of ontologies of a sort commonly called 'presentist'. Each such ontology applies to a particular time. These temporally specified continuant ontologies recognize no diachronic relations. Diachronic facts about conti- 
nuants are rather accounted through relations between entities in ontologies with different time indices, and thus temporal considerations concerning continuants are relegated to the meta-ontological level (Smith and Grenon 2004).

Continuants gain and lose parts over time. It is thus not determinate, given two continuants, whether one is part of the other, because the relations between the two can change from one time to the next. Parthood as a relation between continuants must, therefore, always be indexed to time. Similarly, continuants change in their properties, and it is not determinate across time whether a given continuant has a given property particular inhering in it unless this inherence itself is indexed by time.

This implies, too, that there is no diachronic summation of continuants into larger wholes. Suppose, for example that a sausage has a property particular of being straight at one time and another of being bent at a second time. Summing those entities would make for an aggregate, $S$, which contains the sausage, $s$, its straight shape, $u$, and its bent shape, $v$. But these particular straight and bent shape qualities are entities which do not, because they cannot, co-exist within a single whole - for when would this whole exist? The problem of accounting for an entity having incompatible properties has been dubbed the problem of temporary intrinsics by Lewis (1986). The fourdimensionalist has a solution to this problem: $S$ itself is seen as having two temporal parts, one which is made of $s$ and $u$ and one which is made of $s$ and $v$. $S$ itself is therefore an occurrent. The only way to countenance continuants within a single ontology in which they are allowed to exist at different times is therefore to transform them into occurrents.

The friend of genuine continuants might be tempted by the idea that property particulars are in fact relations to time. It is true that property particulars inhere in their bearers at a time; but the reconstruction of such facts of inherence in terms of relations to time is not satisfactory from an ontological standpoint. Certainly it provides a solution to the problem of logical contradiction by creating pairs of distinct consistent statements $-u$ inheresnow $_{1}$ in $s, v$ inheres-now $w_{2}$ in $s$ - but since we know of no ontological ground to these statements we shall persist in our assumption that this 'solution' is a mere syntactic trick.

In contrast to continuants, occurrents are mereologically determinate over time (indeed over worlds): they have the parts they have as a matter of necessity (Olson, in press). Occurrents exist in time in a way that allows them to be apprehended by a single diachronic ontology (an ontology 
whose purview is extended in time): they exist in time by having parts located at different times. Their existence in time is mediated by that of their temporal parts, which deputize for them. Occurrents have their properties not merely constantly (i.e. at all times at which they occur); they (or their respective temporal parts) have their properties essentially. Thus occurrents do not change in the way continuants change (Mellor 1981). Here again time is a dimension of location. But in contrast to continuants, whose existence is not specific to a time, occurrents have no degree of freedom with respect to time, they are tied essentially to it - this is so whether time itself is an entity or some sort of abstraction over the order of occurrents. For what is distinctive of the realm of occurrents is that there is a temporal order, built out of temporal relations such as earlier than, whose relata exist on a par in spite of the fact that they exist at different times. If time is an abstraction, it is an abstraction over the order of occurrents. And whether or not it is an abstraction, it still allows the occurrents which are located along it to be systematic segmented into arbitrarily large and small temporal parts.

Only entities which are existentially on a par may be summed or aggregated into a whole. We can take, for instance, the sum of the beer and the mug into which it as been poured before Fritz drinks it. But once Fritz has drunk and digested his beer and there remains only the mug, then we may not sum the mug at that time with the no longer existing beer from the earlier time. Surely, we can still sum up Fritz and what remains, if anything, from the beer after it has been digested (some aggregate of scattered molecules that is genidentical to the beer (Lewin 1922)). But it is no longer the beer which we sum with Fritz.

Fourdimensionalism goes hand in hand with a view according to which all the entities which exist co-exist; they all exist on a par. For occurrents, this view is, unproblematic. We can create the sum of all the beer drinking done by Fritz in his life, which is an aggregate of temporally separated entities. In contrast, the foregoing considerations to the effect that there is no single diachronic continuant ontology mean that it is some version of the presentist doctrine propounded by Brentano (1976) and Chisholm (1989) which is appropriate for dealing with continuants. The presentist holds that all entities which exist exist at the present time. Presentism and fourdimensionalism are clearly incompatible. The presentist cannot accept the fourdimensionalist view according to which time is an entity that is stretched out, and that there are many times all of which co-exist in the same way that the many marks on a measuring rod co-exist. Times, both instants and 
intervals, are for the fourdimensionalist parts of time and reality as a whole is a fourdimensional spatiotemporal block universe (Alexander 1920).

It is sometimes said that, when tied to presentism, the doctrine of threedimensionalism is not even formulable (Hawley 2001) since in order to formulate temporal assertions (for example to the effect that continuants persist through time), we need to appeal to time itself, which does not belong to the presentist ontology. This means that in order to be formulated threedimensionalism needs a form of eternalism, which allows the successive tagging of specific times as 'the present' on the basis of some sort of indexicalist view. This means adding to our presentist ontology a further piece - one that does not fit with the rest of the ontology - in order to accommodate time. This is not in itself an objection against realism about continuants, however; it is merely an objection to standard reductionistic versions of this doctrine.

The correlation between two ways of persisting and two incompatible ontologies of time is enough to pose the fundamental problem of the ontology of persistence. Drawing on an intuition of Zemach (1970), one way of rationalizing our view of the inconsistency of the bicategorial view which melds the two ontologies together into one would be as follows: distinct ways of existing generate distinct ontologies. Continuants and occurrents are not simply two categories of entities - or two species of existence - but two radically distinct ways of existing in time. And, if both are to be taken ontologically seriously, then they require distinct and incompatible ontologies.

\subsection{Rejection of Monocategorial Eliminativism}

Contemporary debates on the problem of persistence are standardly formulated in terms of the need for an adjudication between two alternative views of temporal existence. These debates grew out of attempts to account for the persistence of different types of entities through time, but they have turned into a fight over what is the unique way in which all temporal entities persist. This in turn has contributed to the obliteration of the distinction between continuants and occurrents, yielding either eliminativism or one or other form of reconstruction (for example, of continuants as more or less gerrymandered varieties of occurrents). The background motivation is, of course, the widespread tendency on the part of contemporary analytical philosophers to believe that they must maximalize ontological parsimony at all costs. 
We, in contrast, are not concerned by a motivation of this sort. We are interested not just in the theoretical elegance of ontologies but also in their practical utility. We thus prefer to conceive debates about reduction and reconstruction as belonging to the domain of logical representation rather than to ontology properly conceived. When it comes to ontology, and not least to the application of ontology in various practical domains, we see the need to have both ways of persisting. (The medical ontologist needs both Fritz, and Fritz's BAC, and the risings and fallings of Fritz's BAC.) Moreover, in addition to these practical considerations, there is also a theoretical argument to the effect that each of the two just-mentioned views in fact requires the other.

Exclusivistic fourdimensionalism holds (in our terms) that only processes exist, and that continuants are special kinds of process-things (Quine 1960). Time is just another dimension, analogous to the three spatial dimensions, and substantial (threedimensional) entities are analyzed away as worms or fibres within the fourdimensional plenum. Strictly speaking there is for the fourdimensionalist no such thing as Fritz. Rather, there exists within the fourdimensional plenum a continuous succession of processes, which are similar in a Fritzing sort of way.

Exclusivistic threedimensionalist holds (in our terms) that only continuants exist. This view seeks to eliminate or reduce events and processes by conceiving all reference to such entities as a mere façon de parler about what is acceptable threedimensionalistically. Talk of occurrents is cashed out, logically, as talk of continuants and the properties ('states') inhering in them at successive points in time. Time itself is an abstraction over statements concerning these.

For us, both varieties of reduction are unacceptable: for processes to stand in the mentioned similarity relation is precisely for them to be a part of Fritz's life. Conversely, there is more to dynamic reality than a mere succession of snapshots along a temporal continuum and more to change than mere discrepancies along this continuum.

At the same time, however, we accept that both varieties of reduction bring important contributions. Fourdimensionalism brings the signal benefit, from the point of view of rigorous ontology, that its ontology is mereologically determinate. This means that, given any $a$ and any $b$, it is always determinate whether $a$ is part of, or overlaps with, or is disjoint from, or is itself a whole including $b$. Indeed, we hold that fourdimensionalism is right in everything it says, but only when it is making assertions about those entities - precisely the fourdimensional ones - which fall properly within its 
purview. The fourdimensionalist thus does indeed offer, from the standpoint of realist perspectivalism, one veridical perspective on reality: the perspective which apprehends processes and the spatiotemporal regions at which they are located. But other, complementary perspectives are required also: namely those threedimensional perspectives which capture, for given instants of time, the substances (and other continuant entities) which exist at and endure through those times. And then not only is it the case that each such instantaneous threedimensionalist ontology also enjoys the same nice feature that it is mereologically determinate, but also that this same feature is preserved when we embrace both the four- and the threedimensional ontologies simultaneously.

The main problem with threedimensionalism, when rigorously (which means, roughly, presentistically) conceived, is that it leaves no room for time. The main problem with fourdimensionalism is that it provides no account of those demarcations in reality which we discover to be so important when we need to understand, for example, the facts of biology (associative mating, disease transmission, host-pathogen interaction) - it provides no counterpart of the joints we seem to find in the world around us as we encounter organisms and cells and molecules. Occurrents merge with each other in unconstrainedly kaleidoscopic ways to make larger and smaller perduring wholes. Again: putative unitary occurrents are from the fourdimensionalist perspective nothing more than fiat products of the sort of gerrymandering alluded to by Quine (1960, §36). Not only can occurrents be segmented at will into temporal parts; they can also be summed without restriction to form wholes which ride roughshod over the natural joints in reality (Varzi 2003).

Neither of the two views, then, is sufficient by itself. But when merged together in a single ontology, they are inconsistent. The solution to this quandary consists in the recognition of the fact that the two views are complementary. Occurrents are precisely the entities we need in order to obtain a temporal line which can be used to index, and provide an ordering for, our plurality of time-specific presentistic ontologies of continuants. The occurrentist view, on the other hand, is in need of continuants as the bearers of its processes, and as that which (through their spatial and temporal boundaries) provide the support for those segmentations of reality at its joints which yield up Fritz and his grandfather. 


\section{Ontological Pluralism}

What is the picture so far? There is a need for ontologies of two distinct types, one for continuants, the other for occurrents. For the former, in fact, we need a multiplicity of time-specific ontologies, for the latter one ontology suffices, which can be assumed to encompass all of time. That continuants need to be dealt with at a time, and thus through a plurality of timeindexed ontologies, does not mean that their existence is confined to one time only - as if they would be replaced by a succession of instantaneous slices. Ontologies of continuants are like snapshots. They yield views of continuants at a time, but these continuants pre- and post-exist such snapshots. We thus reject a strong presentist solution, and also reject approaches such as Chisholm's view of continuants as entia successiva and constituted by a succession of momentary entities (entia per se). It is ontologies in the realm of continuants which are tied to time, not the continuant entities they recognize. The very same continuants reappear in a multiplicity of numerically distinct ontologies, as the very same Fritz can appear in a succession of distinct snapshots. The question then remains as to how, given such a framework, we are to go about unifying the two sorts of ontologies.

The first clue is that changes in continuants are themselves occurrents. They are processes which happen to continuants. There is, then, a relation between a continuant and an occurrent when the latter is happening in or to the former. Each continuant participates in a multiplicity of occurrents and each occurrent involves or has as participants one or more continuants.

For continuants, there is no temporal mereology (no mereology across time), but only a temporalized mereology (a mereology at a time). Occurrents, on the other hand, can be summed across time. This brings us to the major link between continuants and occurrents. We can define the life of a continuant as the aggregate of the occurrents in which it participates. This definition respects our use of this term in our discussion of Fritz, above. It might be thought that this is a dangerous strategy, because the demarcation of occurrents is wild and unconstrained and so is their aggregation. It is here that we most clearly see at work the interdependency and complementarity of the two sorts of ontologies. It is continuants that, by persisting identically through time and through their participating relations to occurrents, provide for the needed individuation of occurrents (a walk to the beer garden is a walk of somebody in particular to somewhere in particular). 
Clean joints in the ontology of processes are provided through the wellboundedness of the temporally extended entities which are their participants.

But continuants change; in particular they change mereologically. How, then, can the continuant ontologies provide for the needed demarcations in the realm of occurrents? Consider, for instance, the changes that occur in an organism such as Fritz, who is constantly exchanging matter with the outside world. It is precisely in order to do justice to this fact that a framework of numerically distinct ontologies is needed, in each of which we once again enjoy the single benefit that the entities recognized are mereologically determinate. Temporal indexation of statements expressing mereological relations among continuants is achieved via reference to these ontologies.

Qualitative changes in continuants, too, can yield non-gerrymandered demarcations within the realm of occurrents: property particulars come into being and they go out of existence. But property particulars may also themselves change; thus Fritz's BAC changes from low to high and his nose changes from pink to red. It is then not simply the substance or substantial entity (Fritz, his nose) which changes, but rather the corresponding quality. That is, it is not simply that the nose has successively the quality of being pink and then of being red. Rather, the nose has all along its quality of being coloured and it is this quality which changes. Fritz's BAC, in the same way, endures through time, but it changes over time in its specific determinates. Colour and BAC are determinables (Johnson 1921). Changes in such determinables come out as successions of shorter-lived determinate qualities. The determinable endures through these changes. Qualities too have a life and participate directly in processes and indirectly in the processes in which their bearers participate (Smith and Grenon 2004).

What about instantaneous entities? Do they belong to an ontology of continuants or to an ontology of occurrents? The answer is that there are instantaneous entities of different categories on both sides of this divide, which participate in the corresponding ways of being in time. Instantaneous entities which fall within an ontology of continuants might be property particulars which, per accidens, endure only instantaneously - for instance, the property-particular enjoyed momentarily by Fritz, whose temperature is rising continuously, of having a temperature of $93^{\circ}$. Instantaneous entities which fall within an ontology of occurrents are, for example, the beginnings and endings of occurrents. Mereological relations are, at a certain level of generality, category preserving. This means that the parts of an 
entity in the category of substantials are always themselves substantial entities and similarly for entities in other categories. Similarly, each of our two sorts of ontologies is mereologically cumulative and dissective (Varzi, to appear). In other words, belonging to an ontology of a given sort is a property which is preserved under mereological relations. The parts of occurrents are occurrents; the parts of continuants are continuants. Thus no instantaneous temporal part of any occurrent falls within the scope of an ontology of continuants and no instantaneous continuant falls within the scope of an ontology of occurrents. In particular, space is not an instantaneous slice of spacetime.

Finally, processes are dependent on their bearers. But in which sense are they dependent? If processes are dependent on continuants in the way that qualities are dependent on their substantial bearers, then this contradicts the claim that eternalism is true for what concerns the realm of occurrents. For if this were the case, then occurrents would cease to exist with their participants. The solution to this puzzle lies, again, in ontological pluralism. There would be a contradiction if processes and their bearers were in the same ontology. Processes co-exist (and thus allow unrestricted mereological summation) over the largest span of time there is. To each of the points of time within this span there can correspond a temporally indexed ontology of continuants. Dependence, too, is temporally indexed and may thus hold simpliciter at these times. This allows us to solve a problem that arises for the non-pluralist presentist, namely the problem of truth-makers for propositions about the past or for propositions involving putative reference to objects no longer existing, such as the proposition that Fritz is taller than Socrates. For even though Socrates no longer exists when Fritz exists and thus there may be no direct relations between them, their respective lives, on the side of occurrents, do indeed co-exist in the relevant sense (Grenon and Smith 2004). The height of Fritz and the height of Socrates are in the same boat as their respective bearers: they do not co-exist. But their lives - the sums of all processes in which these heights, respectively, participate - do co-exist in the relevant sense. We may thus account for the alleged facts of comparison between Socrates and Fritz and for a variety of other problematic transtemporal relations on the continuant side via links between ontologies of the two sorts. 


\section{Conclusion}

We started out from an account of persistence in time which fits common sense in recognizing both continuants and occurrents. We claimed that a simple treatment in terms of a bicategorial ontology does not work because it employs contradictory accounts of the nature of being in time. At the same time we rejected eliminativism, since both continuantism and occurrentism suffer from critical shortcomings, which become especially conspicious when we attempt to apply ontologies to real-world problems. We suggested instead a form of ontological pluralism: a combination of threedimensionalist and fourdimensionalist perspectives, which are mutually incompatible views of reality only when embraced together within a single ontology. The ontological perspectivalism that is implied here consists in the view that any given domain of reality can be viewed from a number of different ontological perspectives, all of which can have equal claim to veridicality. This does not mean that any and every perspective is equally veridical. For our moderate pluralism is of course constrained by realism - by the view that there is a way reality is, independent of the perspectives (languages, theories, cultures, measuring instruments, ontologies) which we human beings adopt.

\section{Acknowledgements}

This paper was written with financial support from the Wolfgang Paul Program of the Alexander von Humboldt Foundation, the European Union Network of Excellence on Medical Informatics and Semantic Data Mining, the Volkswagen Foundation project Forms of Life, and the National Institutes of Health through the NIH Roadmap for Medical Research Grant 1 U 54 HG004028. Thanks go to Robert Arp and Frederic Tremblay for their helpful comments.

\section{REFERENCES}

Alexander, S. 1920 Space, Time and Deity, London: Macmillan.

Bittner, T. and Smith, B 2003 "A Theory of Granular Partitions”, in Duckham, Goodchild and Worboys (eds.), Foundations of Geographic Information Science, 
London: Taylor \& Francis, 117-151.

Brentano, F. 1976 Philosophical Investigations on Space, Time and the Continuum, trans. by B. Smith, London: Croom Helm, 1987.

Chisholm, R.M. 1989 On Metaphysics, Minneapolis: University of Minnesota Press.

Grenon, P., Smith B., and Goldberg, L. 2004 "Biodynamic Ontology”, in D.M. Pisanelli (ed.), Ontologies in Medicine, Amsterdam: IOS Press, 20-38.

Hawley, K. 2001 How Things Persist, Oxford: Clarendon Press.

Ingarden, R. 1964 Time and Modes of Being, Springfield, IL: Charles C. Thomas.

Johnson, W.E. 1921 Logic, Part I, Cambridge: University Press.

Lewin, K. 1922 Der Begriff der Genese in Physik, Biologie und Entwicklungsgeschichte. Eine Untersuchung zur vergleichenden Wissenschaftslehre, Berlin: Borntraeger.

Lewis, D. 1986 On the Plurality of Worlds, Oxford: Blackwell.

Lowe, E.J. 2002 A Survey of Metaphysics, Oxford: Oxford University Press.

Mellor, D.H. 1981 Real Time, Cambridge: Cambridge University Press.

Merricks, T. 1995 "On the Incompatibility of Enduring and Perduring Entities”, Mind, 104, 523-31.

Olson, E.T. (in press) “The Paradox of Increase”, The Monist.

Quine, W.V.O. 1960 Word and Object, Cambridge, MA: The MIT Press.

Sider, T. 2001 Four-Dimensionalism: An Ontology of Persistence and Time, Oxford: Clarendon Press.

Smith, B. 1990 “Characteristica Universalis”, in K. Mulligan (ed.), Language, Truth and Ontology (Philosophical Studies Series), Dordrecht/Boston/Lancaster: Kluwer, 50-81.

- 1997 "On Substances, Accidents and Universals: In Defence of a Constituent Ontology”, Philosophical Papers, 26, 105-127.

Smith, B. and Brogaard, B. 2003 “A Unified Theory of Truth and Reference”, Logique et Analyse, 169-170, 49-93.

Smith, B. and Grenon, P. 2004 "The Cornucopia of Formal-Ontological Relations", Dialectica, 58:3, 279-296.

van Inwagen, P. 2000 “Temporal Parts and Identity Across Time”, The Monist, 83:3, 43759. 
Varzi, A. 2003 "Perdurantism, Universalism and Quantifiers”, Australasian Journal of Philosophy, 81, 208-15.

- (to appear) "Spatial Reasoning and Ontology: Parts, Wholes, and Locations", in Aiello, Pratt-Hartmann, and van Benthem (eds.), The Logic of Space, Dordrecht: Kluwer.

Zemach, E. 1970 “Four Ontologies”, The Journal of Philosophy, 47, 231-247. 